\title{
IRREDUCIBLE CHARACTERS OF SMALL DEGREE OF THE UNITRIANGULAR GROUP
}

\author{
Martin Marjoram
}

We let $q$ be a power of some prime $p$ and for all $n \in \mathbb{N}$, we let $U_{n}(q)$ be the set of $n \times n$ matrices over $\mathbf{F}_{q}$ which are lower-triangular and have every diagonal entry equal to $1 . U_{n}(q)$ is called the unitriangular group of degree $n$ over $\mathbf{F}_{q} \cdot U_{n}(q)$ is a Sylow $p$ subgroup of $\mathrm{GL}_{n}(q)$, the general linear group of invertible $n \times n$ matrices over $\mathbf{F}_{q}$. For any finite group $G$, we will write $\operatorname{Irr}(G)$ for the set of irreducible complex characters of $G$. Isaacs [2] has shown that every element of $\operatorname{Irr}\left(U_{n}(q)\right)$ has degree a power of $q$. Using this result of Isaacs, a theorem of Huppert [1] for the case where $q=p$ can be extended to prove that

$$
\left\{\Gamma(1): \Gamma \in \operatorname{Irr}\left(U_{n}(q)\right)\right\}=\left\{q^{a}: 0 \leq a \leq \frac{n(n-1)}{2}\right\} .
$$

In [4], the author enumerated the irreducible complex characters of $U_{2 m}(q)$ having each of the three highest degrees, as well as the irreducible complex characters of $U_{2 m-1}(q)$ of highest degree. In this paper, we consider the elements of $\operatorname{Irr}\left(U_{n}(q)\right)$ having each of the three lowest degrees, namely $1, q$, and $q^{2}$. As the underlying field will remain fixed throughout, we will write $U_{n}$ in place of $U_{n}(q)$.

It is easy to see that the commutator subgroup $U_{n}^{\prime}$ of $U_{n}$ is given by

$$
U_{n}^{\prime}=\left\{\left(a_{i j}\right) \in U_{n}: a_{i, i-1}=0 \text { for } 2 \leq i \leq n\right\} .
$$

We let $V_{n, 1}=U_{n} / U_{n}^{\prime}$. Then the elements of $\operatorname{Irr}\left(U_{n}\right)$ of degree 1 are obtained by extending the irreducible characters of $V_{n, 1}$ to $U_{n}$. 
As $V_{n, 1}$ is an (elementary) abelian group of order $q^{n-1}$, this means that there are precisely $q^{n-1}$ irreducible characters of $U_{n}$ of degree 1. We will show that the irreducible characters of $U_{n}$ of degrees $q$ and $q^{2}$ may be obtained by extending characters of analogous factor groups and we will count the number having each degree.

For $0 \leq t \leq n-1$, we define

$$
W_{n, t}=\left\{\left(a_{i j}\right) \in U_{n}: a_{i j}=0 \text { if } 1<i-j \leq t\right\} .
$$

Thus, for example, $W_{n, 0}=U_{n}, W_{n, 1}=U_{n}^{\prime}, W_{n, 3}=U_{n}^{\prime \prime}$, and $W_{n, n-1}=I$. Each $W_{n, t}$ is a normal subgroup of $U_{n}$. For $0 \leq t \leq$ $n-1$ we define

$$
V_{n, t}=U_{n} / W_{n, t} .
$$

Thus the elements of $V_{n, t}$ may be identified with lower-triangular $n \times n$ matrices over $\mathbf{F}_{q}$ with 1's along the diagonal and for which we only consider the entries on the main diagonal and on the $t$ diagonals below the main diagonal. We will write $\tilde{A}$ for the elements $A W_{n, t}$ of $V_{n, t}$.

For $1 \leq s \leq n$, we define

$$
\begin{aligned}
N_{n, s} & =\left\{\left(a_{i j}\right) \in U_{n}: a_{i j}=0 \text { for } i>j \text { if either } i \leq s \text { or } j>s\right\} \\
& =\left\{\left(\begin{array}{cc}
I_{s} & 0 \\
C & I_{n-s}
\end{array}\right): C \in M_{n-s, s}\left(\mathbf{F}_{q}\right)\right\}
\end{aligned}
$$

and

$$
\begin{aligned}
H_{n, s} & =\left\{\left(a_{i j}\right) \in U_{n}: a_{i j}=0 \text { for } i>j \text { where } i>s \text { and } j \leq s\right\} \\
& =\left\{\left(\begin{array}{cc}
A & 0 \\
0 & B
\end{array}\right): A \in U_{s}, B \in U_{n-s}\right\} .
\end{aligned}
$$

For all $s, N_{n, s}$ is a normal abelian subgroup of $U_{n}$ and $H_{n, s}$ is a subgroup of $U_{n}$ which complements $N_{n, s}$, i.e. $N_{n, s} \cap H_{n, s}=I$ and $U=N_{n, s} H_{n, s}$. Usually we write the elements of $H_{n, s}$ as $(A, B)$ instead of $\left(\begin{array}{cc}A & 0 \\ 0 & B\end{array}\right)$. 
We will make extensive use of the following lemma. It follows partly from a theorem of Gallagher, while the rest can be deduced from standard Clifford Theory. (See Corollary 6.17 and Problem 6.18 of [3].) The proof is straightforward and we omit it.

Lemma 1. We let $G$ be a finite group, $N$ a normal abelian subgroup of $G$, and $H$ a subgroup of $G$ which complements $N$. We let $\lambda$ be an element of $\operatorname{Irr}(N)$ and we let $T_{G}(\lambda)$ be the stabilizer of $\lambda$ in $G$, i.e.

$$
T_{G}(\lambda)=\left\{g \in G: \lambda^{g}=\lambda\right\},
$$

where $\lambda^{g} \in \operatorname{Irr}(N), \lambda^{g}(n)=\lambda\left(g^{-1} n g\right)$ for all $n \in N$. We let $S_{G}(\lambda)=T_{G}(\lambda) \cap H \cong T_{G}(\lambda) / N$. Note that $T_{G}(\lambda)=S_{G}(\lambda) N$. We define a character $\bar{\lambda}$ of $T_{G}(\lambda)$ by $\bar{\lambda}(h n)=\lambda(n)$ for all $h$ in $S_{G}(\lambda)$ and all $n$ in $N$. Then for each irreducible character $\Psi$ of $S_{G}(\lambda)$, $(\bar{\lambda} \Psi)^{G}$ is an element of $\operatorname{Irr}(G)$, distinct for distinct $\Psi$ 's, and

$$
\lambda^{G}=\sum_{\Psi \in \operatorname{Irr}\left(S_{G}(\lambda)\right)} \Psi(1)(\bar{\lambda} \Psi)^{G} .
$$

Starting instead with an irreducible character $\Gamma$ of $G$, if $\Gamma$ lies over $\lambda \in \operatorname{Irr}(N)$, then there exists an irreducible character $\Psi$ of $S_{G}(\lambda)$ such that $\lambda$ occurs with multiplicity $\Psi(1)$ in $\left.\Gamma\right|_{N}$ and

$$
\Gamma(1)=\Psi(1) \frac{|H|}{\left|S_{G}(\lambda)\right|} .
$$

For $1 \leq s \leq n$ and for every $s \times(n-s)$ matrix $D$ over $\mathbf{F}_{q}$, we define $\Lambda_{D}: N_{n, s} \rightarrow \mathbb{C}$ by

$$
\Lambda_{D}\left(\left(\begin{array}{cc}
I_{s} & 0 \\
C & I_{n-s}
\end{array}\right)\right)=\omega^{T(\operatorname{tr}(C D))}
$$

where $\omega$ is a primitive $p^{\text {th }}$ root of unity in $\mathbb{C}, T: \mathbf{F}_{q} \rightarrow \mathbf{F}_{p}$ is the usual trace mapping from an extension field into the ground field, $\operatorname{tr}$ denotes the trace of a square matrix, and we identify the elements of $\mathbf{F}_{p}$ with the integers $0,1, \ldots, p-1$. 
Lemma 2. For $1 \leq s \leq n, \operatorname{Irr}\left(N_{n, s}\right)=\left\{\Lambda_{D}: D \in M_{s, n-s}\right\}$ and $\Lambda_{D_{1}}=\Lambda_{D_{2}}$ if and only if $D_{1}=D_{2}$.

Proof: It is easy to check that $\Lambda_{D}$ is a degree 1 representation, and so an irreducible character, of $N_{n, s} . N_{n, s}$ is abelian with $q^{s(n-s)}$ elements, so the number of $\Lambda_{D}$ 's is equal to the number of irreducible characters of $N_{n, s}$. The proof will be complete if we show that $\Lambda_{D_{1}}=\Lambda_{D_{2}}$ implies that $D_{1}=D_{2}$. Now,

$$
\begin{aligned}
\Lambda_{D_{1}}=\Lambda_{D_{2}} & \Rightarrow \omega^{T\left(\operatorname{tr}\left(C D_{1}\right)\right)}=\omega^{T\left(\operatorname{tr}\left(C D_{2}\right)\right)} \text { for all } C \in M_{n-s, s}\left(\mathbf{F}_{q}\right) \\
& \Rightarrow T\left(\operatorname{tr}\left(C\left(D_{1}-D_{2}\right)\right)\right)=0 \text { for all } C \in M_{n-s, s}\left(\mathbf{F}_{q}\right) \\
& \Rightarrow \operatorname{tr}\left(C\left(D_{1}-D_{2}\right)\right)=0 \text { for all } C \in M_{n-s, s}\left(\mathbf{F}_{q}\right)
\end{aligned}
$$

where the last implication follows from the well-known fact that $T: \mathbf{F}_{q} \rightarrow \mathbf{F}_{p}$ is non-zero.

But the last condition above implies that $D_{1}-D_{2}=0$. For suppose the $(i, j)$-entry of $D_{1}-D_{2}$ is non-zero. Then we can let $C=E_{j i}$, the $(n-s) \times s$ matrix with 1 in the $(j, i)-$ position and zeros elsewhere. It is clear that for this choice of $C$, $\operatorname{tr}\left(C\left(D_{1}-D_{2}\right)\right)$ is equal to the $(i, j)$-entry of $D_{1}-D_{2}$, and so is not equal to zero, a contradiction. Thus $\Lambda_{D_{1}}=\Lambda_{D_{2}}$ implies that $D_{1}=D_{2}$ and the proof is complete.

For $1 \leq s \leq n$, we can now apply the result from Lemma 1 to the normal abelian subgroup $N_{n, s}$ of $U_{n}$ and its complement $H_{n, s}$. For all elements $D$ of $M_{s, n-s}$, we let $S(D)=S_{U_{n}}\left(\Lambda_{D}\right)$.

Lemma 3. Let $\Lambda_{D}$ be an element of $\operatorname{Irr}\left(N_{n, s}\right)$ for some value of $s$. Then

$$
S(D)=\left\{(A, B) \in H_{n, s}: A D B^{-1}=D\right\} .
$$


Proof: Let $(A, B)$ be any element of $H_{n, s}$. Then

$$
\begin{aligned}
& \Lambda_{D}^{(A, B)}\left(\left(\begin{array}{cc}
I & 0 \\
C & I
\end{array}\right)\right)=\Lambda_{D}\left(\left(\begin{array}{cc}
A & 0 \\
0 & B
\end{array}\right)^{-1}\left(\begin{array}{cc}
I & 0 \\
C & I
\end{array}\right)\left(\begin{array}{cc}
A & 0 \\
0 & B
\end{array}\right)\right) \\
& =\Lambda_{D}\left(\left(\begin{array}{cc}
I & 0 \\
B^{-1} C A & I
\end{array}\right)\right) \\
& =\omega^{T\left(\operatorname{tr}\left(B^{-1} C A D\right)\right)} \\
& =\omega^{T\left(\operatorname{tr}\left(C A D B^{-1}\right)\right)} \\
& =\Lambda_{A D B^{-1}}\left(\left(\begin{array}{ll}
I & 0 \\
C & I
\end{array}\right)\right)
\end{aligned}
$$

for all elements $\left(\begin{array}{cc}I & 0 \\ C & I\end{array}\right)$ of $N_{n, s}$. Thus $\Lambda_{D}^{(A, B)}=\Lambda_{A D B^{-1}}$ and

$$
\begin{aligned}
S(D) & =\left\{(A, B) \in H_{n, s}: \Lambda_{D}^{(A, B)}=\Lambda_{D}\right\} \\
& =\left\{(A, B) \in H_{n, s}: \Lambda_{A D B^{-1}}=\Lambda_{D}\right\} \\
& =\left\{(A, B) \in H_{n, s}: A D B^{-1}=D\right\} .
\end{aligned}
$$

It follows from the proof above that $H_{n, s}$ acts on $\operatorname{Irr}\left(N_{n, s}\right)$ via the action $(A, B): \Lambda_{D} \mapsto \Lambda_{A D B^{-1}}$. We let $\mathcal{O}\left(\Lambda_{D}\right)$ be the orbit of $\Lambda_{D}$ under this action. Of course every element $\Lambda_{D^{\prime}}$ of $\mathcal{O}\left(\Lambda_{D}\right)$ lies under precisely the same irreducible characters of $U_{n}$ as $\Lambda_{D}$ while $S\left(D^{\prime}\right) \cong S(D)$.

Lemma 4. Let $\Gamma \in \operatorname{Irr}\left(U_{n}\right)$ and choose $t$ maximal such that $W_{n, t+1} \leq \operatorname{ker}(\Gamma)$ but $W_{n, t} \not \mathbf{k e r}(\Gamma)$. Then there exists $Y \in W_{n, t}$ such that $Y \notin \operatorname{ker}(\Gamma)$ and $Y$ has precisely one non-zero entry below the main diagonal.

Proof: Let $X=\left(x_{i j}\right)$ be an element of $W_{n, t}-\operatorname{ker}(\Gamma)$. We define

$$
Z=I_{n}-\sum_{l=t+2}^{n} x_{l, l-t-1} E_{l, l-t-1}
$$


We claim that $Z \notin \operatorname{ker}(\Gamma)$. For if $f$ is the representation of $U$ which affords $\Gamma$, then $f(Z X)=I$ because $Z X \in W_{n, t+1}$. Thus $f(Z)=f(X)^{-1} \neq I$. We note that

$$
\begin{aligned}
Z= & \left(I-x_{t+2,1} E_{t+2,1}\right)\left(I-x_{t+3,2} E_{t+3,2}\right) \ldots \\
& \left(I-x_{n, n-t-1} E_{n, n-t-1}\right) .
\end{aligned}
$$

If every term in this product were an element of $\operatorname{ker}(f)$, then $Z$ would also be an element of $\operatorname{ker}(f)$. Thus for at least one choice of $k$ satisfying $t+2 \leq k \leq n$,

$$
I-x_{k, k-t-1} E_{k, k-t-1} \notin \operatorname{ker}(\Gamma),
$$

as required.

Theorem 5. Take $\Gamma \in \operatorname{Irr}\left(U_{n}\right)$ and choose $t$ maximal such that $W_{n, t+1} \leq \operatorname{ker}(\Gamma)$ but $W_{n, t} \not \mathbf{k e r}(\Gamma)$. Then $\Gamma(1) \geq q^{t}$.

Proof: We choose $Y=I+\lambda E_{k, k-t-1}$ not in $\operatorname{ker}(\Gamma)$. We note that $Y$ is an element of $N_{n, k-1}$. Thus there is at least one element $\Lambda_{D}$ of $\operatorname{Irr}\left(N_{n, k-1}\right)$ for which $Y$ is not an element of $\operatorname{ker}\left(\Lambda_{D}\right)$, i.e. $\operatorname{tr} \lambda E_{1, k-t-1} D \neq 0$, where $D$ is a $(k-1) \times(n-k+1)$ matrix over $\mathbf{F}_{q}$. If $D=\left(d_{i j}\right)$, then it follows that $d_{k-t-1,1} \neq 0$. We let $\alpha=d_{b 1}$ be the first non-zero entry in column 1 of $D$. Then $b \leq k-t-1$. (In fact it must be the case that $b=k-t-1$, but we do not need to prove this.) We let

$$
A=I-\sum_{l=b+1}^{n} \frac{d_{l 1}}{\alpha} E_{l b} .
$$

We set $D^{\prime}=A D$. Then $\Lambda_{D^{\prime}}$ is an element of $\mathcal{O}\left(\Lambda_{D}\right)$ and

$$
\begin{aligned}
D^{\prime} & =A D \\
& =\left(I-\sum_{l=b+1}^{n} \frac{d_{l 1}}{\alpha} E_{l b}\right)\left(\alpha E_{b 1}+\sum_{l=b+1}^{n} d_{l 1} E_{l 1}+\sum_{\substack{1 \leq i \leq k-1 \\
2 \leq j \leq n-k+1}} d_{i j} E_{i j}\right) \\
& =\alpha E_{b 1}+\sum_{\substack{1 \leq i \leq k-1 \\
2 \leq j \leq n-k+1}} d_{i j}^{\prime} E_{i j},
\end{aligned}
$$


for some $d_{i j}^{\prime}$ 's in $\mathbf{F}_{q}$. Thus $D^{\prime}$ has precisely one non-zero entry in column 1 , namely $\alpha$ in the $(b, 1)$-position.

Now we have

$$
S(D) \cong S\left(D^{\prime}\right)=\left\{(A, B) \in H_{n, k-1}: A D^{\prime} B^{-1}=D^{\prime}\right\} .
$$

Writing $A=\left(a_{i j}\right)$, the equation $A D^{\prime}=D^{\prime} B$ gives us relations which the $k-1-b$ entries $a_{b+1, b}, a_{b+2, b}, \ldots, a_{k-1, b}$ must satisfy. Thus

$$
\begin{aligned}
|S(D)|=\left|S\left(D^{\prime}\right)\right| & \leq \frac{\left|H_{n, k-1}\right|}{q^{k-1-b}} \\
& \leq \frac{\left|H_{n, k-1}\right|}{q^{k-1-(k-t-1)}} \\
& =\frac{\left|H_{n, k-1}\right|}{q^{t}} .
\end{aligned}
$$

By Lemma 1, there exists $\Psi \in \operatorname{Irr}(S(D))$ such that

$$
\begin{aligned}
\Gamma(1) & =\Psi(1) \frac{\left|H_{n, k-1}\right|}{|S(D)|} \\
& \geq \frac{\left|H_{n, k-1}\right|}{\left|H_{n, k-1}\right| / q^{t}} \\
& =q^{t} .
\end{aligned}
$$

Definition: For a finite group $G$ and a positive integer $l$, we define $C(G, l)$ to be the number of irreducible characters of $G$ having degree $l$.

Corollary 6. Choose $t$ such that $1 \leq t \leq n-1$. Then for $0 \leq a \leq t-1$, we have

$$
C\left(U_{n}, q^{a}\right)=C\left(V_{n, t}, q^{a}\right)
$$

Proof: If $\Gamma$ is an element of $\operatorname{Irr}\left(U_{n}\right)$ such that $\Gamma(1) \leq q^{t-1}$, then by Theorem $5 W_{n, t} \leq \operatorname{ker}(\Gamma)$. Thus $\Gamma$ is an element of $\operatorname{Irr}\left(V_{n, t}\right)$. Conversely, given any element $\Theta$ of $\operatorname{Irr}\left(V_{n, t}\right)$, we can extend $\Theta$ to 
an element of $\operatorname{Irr}\left(U_{n}\right)$ by letting $\Theta(A)=\Theta(I)$ for all elements $A$ of $W_{n, t}$.

To find the number of irreducible characters of $U_{n}$ of degrees $q$ and $q^{2}$, we need only calculate $C\left(V_{n, 2}, q\right)$ and $C\left(V_{n, 3}, q^{2}\right)$. While our main interest in $V_{n, 2}$ is in the degree $q$ characters, it turns out that for this group there is a simple closed formula describing the number of irreducible characters having any degree. The formulas we derive involve binomial coefficients. We adopt the conventions that $\left(\begin{array}{l}0 \\ 0\end{array}\right)=1$ and $\left(\begin{array}{l}s \\ t\end{array}\right)=0$ whenever $s<t$.

Theorem 7. $\left\{\Theta(1): \Theta \in \operatorname{Irr}\left(V_{n, 2}\right)\right\}=\left\{q^{a}: 0 \leq a \leq \frac{n-1}{2}\right\}$ and, for all values of $a$ in this range,

$$
C\left(V_{n, 2}, q^{a}\right)=\left(\begin{array}{c}
n-a-1 \\
a
\end{array}\right) q^{n-a-2}(q-1)^{a}+\left(\begin{array}{c}
n-a-2 \\
a
\end{array}\right) q^{n-a-2}(q-1)^{a+1} .
$$

Proof: We proceed by induction on $n$. $V_{2,2}=U_{2}$ has $q$ irreducible characters, all of degree 1 , as the theorem states. $V_{3,2}=U_{3}$ has $q^{2}$ characters of degree 1 while every other character degree is a power of $q$. Recall that the squares of the degrees of the irreducible characters of a finite group $G$ add up to the order of $G$. It follows that $\left\{\Theta(1): \Theta \in \operatorname{Irr}\left(V_{3,2}\right)\right\}=\{1, q\}$ and that $C\left(V_{3,2}, q\right)=q-1$, which again is in agreement with the statement of the theorem.

For $n>3$, we let

$$
J_{n}=\left\{\left(a_{i j}\right)+W_{n, 2} \in V_{n, 2}: a_{n, n-2}=a_{n, n-1}=0\right\}
$$

and

$$
K_{n}=\left\{\left(b_{i j}\right)+W_{n, 2} \in V_{n, 2}: b_{i j}=0 \text { for } i \neq n\right\} .
$$

Now $K_{n}$ is a normal abelian subgroup of $V_{n, 2}$, having $J_{n}$ as complement. For all $\alpha$ and $\beta$ in $\mathbf{F}_{q}$, we define $\Delta_{\alpha, \beta}: K_{n} \rightarrow \mathbb{C}$ by

$$
\Delta_{\alpha, \beta}\left(\left(b_{i j}\right)+W_{n, 2}\right)=\omega^{\alpha b_{n, n-2}+\beta b_{n, n-1}} .
$$

It is easy to prove that $\operatorname{Irr}\left(K_{n}\right)=\left\{\Delta_{\alpha, \beta}: \alpha, \beta \in \mathbf{F}_{q}\right\}$ and $\Delta_{\alpha^{\prime}, \beta^{\prime}}=\Delta_{\alpha, \beta}$ if and only if $\alpha^{\prime}=\alpha$ and $\beta^{\prime}=\beta$. Furthermore, for 
any elements $\tilde{A}=\left(a_{i j}\right)+W_{n, 2}$ of $J_{n}$ and $\tilde{B}=\left(b_{i j}\right)+W_{n, 2}$ of $K_{n}$,

$$
\begin{aligned}
\Delta_{\alpha, \beta}^{\tilde{A}}(\tilde{B}) & =\Delta_{\alpha, \beta}^{\tilde{A}}\left(\left(b_{i j}\right)+W_{n, 2}\right) \\
& =\Delta_{\alpha, \beta}\left(A^{-1}\left(b_{i j}\right) A+W_{n, 2}\right) \\
& =\Delta_{\alpha, \beta}\left(I+\left(b_{n, n-2}+b_{n, n-1} a_{n-1, n-2}\right) E_{n, n-2}+\right. \\
& \left.\quad b_{n, n-1} E_{n, n-1}+W_{n, 2}\right) \\
& =\omega^{T\left(\alpha\left(b_{n, n-2}+b_{n, n-1} a_{n-1, n-2}\right)+\beta b_{n, n-1}\right)} \\
& =\omega^{T\left(\alpha b_{n, n-2}+\left(\beta+\alpha a_{n-1, n-2}\right) b_{n, n-1}\right)} \\
& =\Delta_{\alpha,\left(\beta+\alpha a_{n-1, n-2}\right)}(\tilde{B}),
\end{aligned}
$$

i.e. $\Delta_{\alpha, \beta}^{\tilde{A}}=\Delta_{\alpha,(\beta+\alpha \varepsilon)}$, where $\varepsilon=a_{n-1, n-2}$.

We define $S(\alpha, \beta)=S_{V_{n, 2}}\left(\Delta_{\alpha, \beta}\right)$. Then for all non-zero elements $\alpha$ of $\mathbf{F}_{q}$ and all elements $\beta$ of $\mathbf{F}_{q}$,

$$
S(0, \beta)=J_{n} \cong V_{n-1,2}
$$

and

$$
\begin{aligned}
S(\alpha, \beta) & \cong S(\alpha, 0) \\
& =\left\{\left(a_{i j}\right)+W_{n, 2} \in J_{n}: a_{n-1, n-2}=0\right\} \\
& =\left\{\left(a_{i j}\right)+W_{n, 2} \in V_{n, 2}: a_{n-1, n-2}=a_{n, n-1}=a_{n, n-2}=0\right\} \\
& \cong V_{n-2,2} \oplus\left(\mathbf{F}_{q},+\right) .
\end{aligned}
$$

For all non-zero elements $\alpha$ of $\mathbf{F}_{q}$ and all elements $\beta$ of $\mathbf{F}_{q}$, Lemma 1 tells us that

$$
\begin{aligned}
& \left\{\Gamma \in \operatorname{Irr}\left(V_{n, 2}\right):\left\langle\left.\Gamma\right|_{N}, \Delta_{0, \beta}\right\rangle_{N} \neq 0\right\} \\
= & \left\{\left(\bar{\Delta}_{0, \beta} \Psi\right)^{V_{n, 2}}: \Psi \in \operatorname{Irr}(S(0, \beta))\right\}
\end{aligned}
$$

and 


$$
\begin{aligned}
& \left\{\Gamma \in \operatorname{Irr}\left(V_{n, 2}\right):\left\langle\left.\Gamma\right|_{N}, \Delta_{\alpha, \beta}\right\rangle_{N} \neq 0\right\} \\
= & \left\{\Gamma \in \operatorname{Irr}\left(V_{n, 2}\right):\left\langle\left.\Gamma\right|_{N}, \Delta_{\alpha, 0}\right\rangle_{N} \neq 0\right\} \\
= & \left\{\left(\bar{\Delta}_{\alpha, 0} \Psi\right)^{V_{n, 2}}: \Psi \in \operatorname{Irr}(S(\alpha, 0))\right\} .
\end{aligned}
$$

Lemma 1 also implies that, with the same notation as in the sets above,

$$
\left(\bar{\Delta}_{0, \beta} \Psi\right)^{V_{n, 2}}(1)=\Psi(1)
$$

and

$$
\left(\bar{\Delta}_{\alpha, 0} \Psi\right)^{V_{n, 2}}(1)=q \Psi(1) .
$$

Thus for $0 \leq a \leq \frac{n-2-1}{2}+1$,

$$
\begin{aligned}
C\left(V_{n, 2}, q^{a}\right)= & q C\left(V_{n-1,2}, q^{a}\right)+q(q-1) C\left(V_{n-2,2}, q^{a-1}\right) \\
= & q\left(\begin{array}{c}
n-1-a-1 \\
a
\end{array}\right) q^{n-1-a-2}(q-1)^{a}+ \\
& q\left(\begin{array}{c}
n-1-a-2 \\
a
\end{array}\right) q^{n-1-a-2}(q-1)^{a+1}+ \\
& q(q-1)\left(\begin{array}{c}
n-2-(a-1)-1 \\
a-1
\end{array}\right) q^{n-2-(a-1)-2}(q-1)^{a-1}+ \\
& q(q-1)\left(\begin{array}{c}
n-2-(a-1)-2 \\
a-1
\end{array}\right) q^{n-2-(a-1)-2}(q-1)^{a} \\
= & \left(\begin{array}{c}
n-a-2 \\
a
\end{array}\right) q^{n-a-2}(q-1)^{a}+\left(\begin{array}{c}
n-a-3 \\
a
\end{array}\right) q^{n-a-2}(q-1)^{a+1}+ \\
& \left(\begin{array}{c}
n-a-2 \\
a-1
\end{array}\right) q^{n-a-2}(q-1)^{a}+\left(\begin{array}{c}
n-a-3 \\
a-1
\end{array}\right) q^{n-a-2}(q-1)^{a+1} \\
= & \left(\begin{array}{c}
n-a-1 \\
a
\end{array}\right) q^{n-a-2}(q-1)^{a}+\left(\begin{array}{c}
n-a-2 \\
a
\end{array}\right) q^{n-a-2}(q-1)^{a+1} .
\end{aligned}
$$

It seems to be much more difficult to find a formula for $C\left(V_{n, 3}, q^{a}\right)$ which holds for all relevant values of $a$. In this case, we will confine our attention to the characters of degree $q^{2}$ and state without proof our findings in this case.

Theorem 8. We have $C\left(V_{4,3}, q^{2}\right)=q(q-1)$ and for $n \geq 5$,

$$
\begin{aligned}
C\left(V_{n, 3}, q^{2}\right)= & \left(\begin{array}{c}
n-3 \\
2
\end{array}\right) q^{n-4}(q-1)^{2}+\left(\begin{array}{c}
n-4 \\
2
\end{array}\right) q^{n-4}(q-1)^{3}+ \\
& (n-3) q^{n-2}(q-1)+(n-5) q^{n-2}(q-1)^{2} .
\end{aligned}
$$


We can now provide formulae for the number of irreducible characters of $U_{n}$ having the degrees $1, q$ and $q^{2}$.

Theorem 9. For $n \geq 2, U_{n}$ has $q^{n-1}$ irreducible characters of degree 1 . For $n \geq 3, U_{n}$ has $(n-2) q^{n-3}(q-1)+(n-3) q^{n-3}(q-1)^{2}$ irreducible characters of degree $q$. $U_{4}$ has $q(q-1)$ irreducible characters of degree $q^{2}$; for $n \geq 5, U_{n}$ has $\left(\begin{array}{c}n-3 \\ 2\end{array}\right) q^{n-4}(q-1)^{2}+$ $\left(\begin{array}{c}n-4 \\ 2\end{array}\right) q^{n-4}(q-1)^{3}+(n-3) q^{n-2}(q-1)+(n-5) q^{n-2}(q-1)^{2}$ irreducible characters of degree $q^{2}$.

Proof: This follows from Corollary 6, Theorem 7, and Theorem 8.

\section{References}

[1] B. Huppert, A remark on character degrees of some p-groups, Archiv der Math. 59 (1992), 313-318.

[2] I. M. Isaacs, Characters of groups associated with finite algebras, J. Algebra 177 (1995), 708-730.

[3] I. M. Isaacs, Character Theory of Finite Groups. Dover: New York, 1994.

[4] M. Marjoram, Irreducible characters of Sylow p-subgroups of classical groups, Ph. D. thesis (1997), National University of Ireland, Dublin.

Martin Marjoram

Department of Mathematics

University College

Belfield

Dublin 4 